\title{
The Evolution of Future Medicine - WE Medicine - To Meet Unmet Medical Needs
}

\author{
Yung Chi Cheng ${ }^{1 *}$, Peikwen Cheng ${ }^{2}$, Shwu Huey Liư ${ }^{2}$, Wing Lam ${ }^{1}$, Fulan Guan ${ }^{1}$, \\ Rong $\mathrm{Hu}^{1}$ and William Cheng $^{2}$ \\ ${ }^{1}$ Department of pharmacology, Yale School of Medicine, USA \\ ${ }^{2}$ Yiviva Inc., USA
}

ISSN: 2637-773X

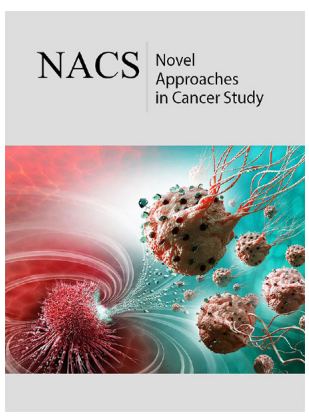

*Corresponding author: Yung Chi Cheng, Department of pharmacology, Yale School of Medicine, Yale University, USA

Submission: 海 October 17, 2019

Published: 制 November 07, 2019

Volume 3 - Issue 5

How to cite this article: Yung Chi C, Peikwen C, Shwu H L, Wing L, Fulan G, Rong H, William C. The Evolution of Future Medicine - We Medicine - To Meet Unmet Medical Needs. Nov Appro in Can Study. 3(5). NACS.000572.2019.

DOI: 10.31031/NACS.2019.03.000572

Copyright@ Yung Chi Cheng, This article is distributed under the terms of the Creative Commons Attribution 4.0 International License, which permits unrestricted use and redistribution provided that the original author and source are credited.

\section{Introduction}

The medicines needed today are not just for the treatment of disease, but for the treatment of the whole person across his or her lifetime - from disease prevention to disease progression, modulating the efficacy and safety of other medicines, and improving quality of life.

Currently many pharmaceutical and biotech companies are focusing primarily on the treatment versus the prevention of disease, with the exception of developing vaccines against infectious agents as preventative measurement. Developing small molecule medicines still largely applies a reductionist approach focused on a particular target and developing selective and potent chemicals aimed at it. The advancement of knowledge and modern technologies has facilitated the reductionist approach and led to the discovery of a few interesting target- oriented drugs with varying degrees of success, however this approach has not been satisfactory for the treatment of more complex, heterogeneous diseases which are often associated with the aging process [1]. It is recognized that the selected target in most cases is not only important for the pathogenesis of disease, but also plays an important role in the normal functions of the body. With highly potent chemicals used on long term basis, toxicity in normal tissues will often evolve. Furthermore, there is heterogeneity of the tissue target phenotype due to the degree of nutrients, including oxygen, and interaction among cells in the tissue [2]. Drugs could have a different impact on cells depending on its target phenotype or other cellular components. The response of targeted cells to drugs could 'be different. This is clearly evident in cancer tissue (Figure 1).

\section{Current Medicine has a Broad Range of Needs}

\begin{tabular}{|l|l|}
\hline Need & Current System \\
\hline Treatment of Disease & Therapeutic Medicine \\
\hline Prevention of Disease & Preventative Medicine \\
\hline $\begin{array}{l}\text { Enhancement of "Quality of Life" of } \\
\text { Patients and "healthy" individuals }\end{array}$ & Functional Medicine \\
\hline Improving use of other medicines & Adjuvant Medicine \\
\hline
\end{tabular}

Figure 1: Current medicine has a broad range of needs.

This target-oriented approach will have limited value for preventative medicines since disease initiation, etiology and progression can be different among different individuals [3]. Additionally, drug resistance or tolerance, as a result of target mutations and/or adaptive metabolic changes, are common issues for a reductionist, target-oriented therapies. Therefore, the current reductionist approach is useful but not sufficient to fulfill more complex, unmet 
clinical needs. A new paradigm should take a holistic, systems biology approach and may require multiple chemicals working on multiple targets within the same tissue or across different tissues. If our current knowledge and understanding of the pathogenesis is sufficient, this approach can be done rationally by combining existing drugs to optimize each other's action. However, this rational approach could be difficult since we do not have an overall knowledge about the dynamic nature of the body's system, including the diseased tissue as well as the drug. An alternative approach is to develop a systems biology approach. Using modern science, we may be able to learn from the wealth of knowledge from experienced-based, traditional botanical medicines. Among all traditional medicines, traditional Chinese medicine (TCM) is one of the most advanced Eastern (E) medicines due to its thousands of years of experience and written history, and has evolved into several branches, including current Chinese medicine, Japan Kampo medicine, Korean Han medicine, Vietnamese medicine etc. (Figure 2).

\section{Target Oriented Cancer Drugs Alone have Their Value AND Limitations}

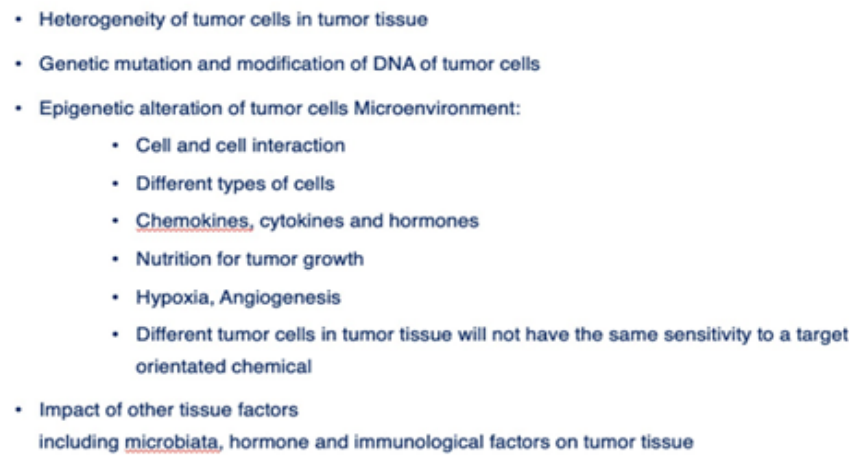

Figure 2: Target oriented cancer drugs alone have their value and limitations.

\section{Traditional Medicines from Around the World $>$ Advanced in Hot Pot of Chinese Medicine}
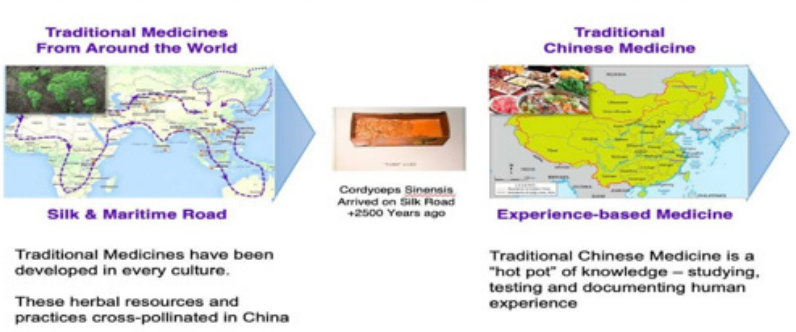

Figure 3: Traditional medicines from around the world $>$ advanced in hot pot of Chinese medicine.

TCM is a melting product of human wisdom - accumulated over thousands of years through the sharing of knowledge with different cultures and the experimentation of different traditional medicines from round the world [4]. This is exemplified by the variety of herbs used currently in TCM still being used by other world cultures (Figure 3). TCM has evolved to treat human health conditions through its own principles and concepts applying more on a macroscopic level and holistic approach. Its effectiveness often requires multiple chemicals to act on different targets or organs. Seven types of herb interactions with respect to their effectiveness was described such as antagonism, potentiation, additive, synergism, etc. The polychemical interactions form the basis for TCM formulas and these principles, while using different terms, are also applied in Western drug formulations and usage (Figure 4).

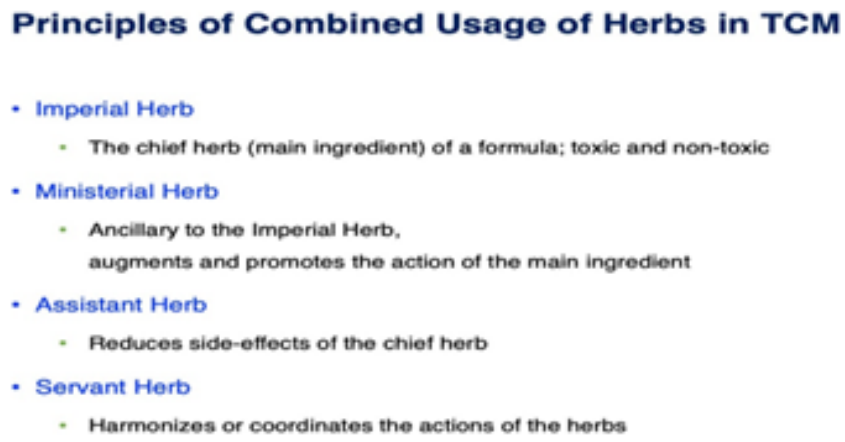

Figure 4: Principles of combined usage of herbs in TCM.

TCM approach is different from but can be highly complementary with current molecular medicines evolved from Western (W) medicine, which focuses more on a microscopic level and single targets to treat diseases. In order to meet unmet future health needs, neither Eastern (E) medicines, exemplified by TCM, nor Western (W) medicines, exemplified by current conventional medicine, is sufficient. However, while seemingly different, we may be experiencing a convergence of these two paradigms particularly in the area of prevention and treatment of aging and chronic diseases and symptoms (Figure 5).

\section{Convergence:}

Western Medicine $><$ Traditional Chinese Medicine

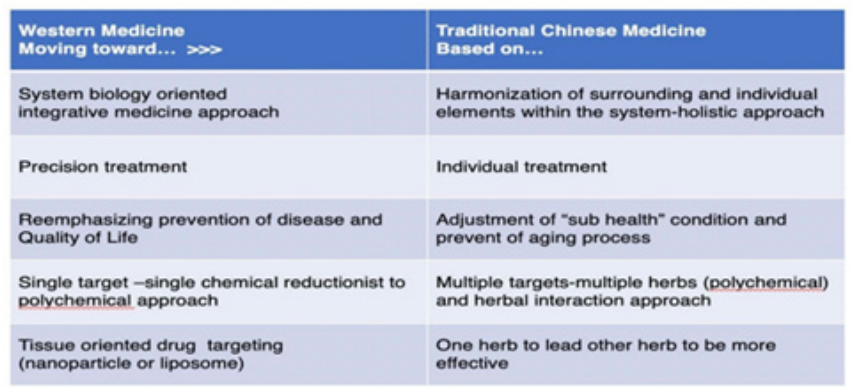

Figure 5: Convergence: western medicine $><$ Traditional Chinese medicine.

The most effective approach for developing future medicine to meet unmet clinic needs should be the melting of "W" and " $E$ " into WE Medicine (Figure 6). For patients, it should be noted that "Medicine is medicine" and future medicines should meet certain requirements for safety, efficacy and quality. In order to modernize botanical medicines into WE Medicine, it necessary to overcome several challenges: 


\section{Evolution of Medicine}

\section{Past $\mathrm{W}=\mathrm{E}$ Present $\mathrm{W} \rightleftharpoons \mathrm{E}$ \\ Future

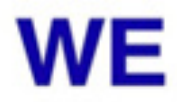

Figure 6: Evolution of medicine.

\section{High quality and consistent manufacturing}

a) No contamination

b) Identification of the right herb to treat specific health conditions should be ensured

c) Growing location and harvesting conditions should be well defined

d) Processing and preservation of the herbs should be standardized

e) Manufacturing process should follow standard operational procedure

f) Quality control to demonstrate batch-to-batch consistency should be developed and used.

Mechanism- based methodology for quality control should be employed currently. The use of chemical fingerprints and chemical marker methodology has limitations if the active compounds for a given clinical indication are not clear.

\section{Evidence-based clinical studies to validate efficacy and safety}

There are several key points:

a. Animal preclinical studies have limitations. If a botanical drug is made following historical usage, no preclinical toxicity study may be necessary before clinical study, as historical information can be leveraged.

b. Information-rich clinical studies including the use of -omics technologies to analyze biomarkers should be incorporated - which could be useful for understanding prognosis, treatment, side-effects and usage.

c. Randomized, double-blind, placebo-controlled, multiregional clinical trials should be planned to validate its efficacy. Modern day acceptable clinical endpoint should be used. Racial differences in response should be carefully evaluated.

d. Chinese pattern diagnosis before and during treatment could be included as a useful method to stratify patients for precision medicine. The quality control of the diagnosis is critical to yield useful information.

\section{Safety assessment of dosage and usage of product}

To ensure the safe use of botanical medicine, Phase 1 studies targeting diseased patients or populations is encouraged. Current use of animals without disease for toxicity study has limited value, as normal animals in current use may handle chemicals differently from diseased individual. Pharmacokinetic studies of herbs should not be required at this current stage because botanicals have multiple chemicals, and their uptake and metabolism in different tissues may play a unique role in the treatment of disease. The pharmacodynamic studies using modern imaging technology could be useful and information rich [5].

\section{Knowledge of interaction of botanicals and other drugs}

TCM, particularly in Asia, and "Western" medicines are often used together for the treatment and prevention of diseases, particularly in treating complex, aging- associated diseases or symptoms. TCM may have an impact on another drug's metabolism and physiological behaviors [6]. Their interaction should be studied, paying attention to the Western medicine with steep dose response curve. The information could help to decide whether the dosage of Western medicine should be adjusted to achieve its optimal activity or avoid toxicity.

\section{Information of botanical drugs mechanisms of action and active compounds}

Understanding the mechanisms of actions could yield information to improve intended usage and discover new usages for botanical drugs. For instance this lab worked on the mechanisms of action of YIV-906 (PHY906), which was initially developed to relieve gastrointestinal side-effects caused by irinotecan. Subsequently in studies, it was discovered that YIV-906 could not only improve safety but also enhance the antitumor action of irinotecan [7]. Through mechanism of action studies, YIV-906 has demonstrated the potential to be a pan-adjuvant for chemotherapy, radiation therapy and immune checkpoint therapy for cancer treatment. The chemicals involved in botanical medicines will also be very useful. It could help to identify and select optimal herbs for a particular formulation for a particular usage. YIV-906 has different pharmacological properties from commercially available HuangQin Tang, the historical formula it referenced, even though it uses the same four herbs. As with biological drugs, the raw materials and manufacturing process can lead to unique preparations that are not bioequivalent [8].

The well-being of the patient is the most important consideration for a physician. The focus on treating a disease, such as cancer, with not much attention to the well-being of the whole patient is not proper. Furthermore, the health condition and psychological behavior in patients in general, could also affect their disease progression [9]. Thus, a systems biology approach integrating a patients micro-scopic/environment and macroscopic/environment should be integrated into future medicines WE medicine is the future. 


\section{Conflict of interest}

Yung-Chi Cheng and Shwu-huey Liu are the co-inventor of YIV906 and co-founder of Yiviva. Peikwen Cheng, William Cheng and Shwu-huey Liu are employees of Yiviva.

\section{References}

1. Corbet C, Feron $O$ (2017) Tumour acidosis: from the passenger to the driver's seat. Nat Rev Cancer 17(10): 577-593.

2. De Palma M, Biziato D, Petrova TV (2017) Microenvironmental regulation of tumour angiogenesis. Nat Rev Cancer 17(8): 457-474.

3. Su X, Yao Z, Li S, Sun H (2016) Synergism of chinese herbal medicine: Illustrated by danshen compound. Evid Based Complement Alternat Med, Article ID:7279361.

4. Lam W, Ren Y, Guan F, Jiang Z, Cheng W, et al. (2018) Mechanism Based Quality Control (MBQC) of herbal products: A case Study YIV-906 (PHY906). Front Pharmacol 9: 1324.
5. Tilton R, Paiva AA, Guan JQ, Marathe R, Jiang Z, et al. (2010) A comprehensive platform for quality control of botanical drugs (Phytomics QC): A case study of Huangqin Tang (HQT) and PHY906. Chin Med 5: 30.

6. Hecht JR (2001) Gastrointestinal toxicity of irinotecan. Oncology (Williston Park) 12 (8 Suppl 6): 72-78.

7. Lam W, Bussom S, Guan F, Jiang Z, Zhang W, et al. (2010) The fourherb chinese medicine PHY906 reduces chemotherapy-induced gastrointestinal toxicity. Sci Transl Med 2(45): 45ra59.

8. Lam W, Jiang Z, Guan F, Hu R, Liu SH, et al. (2014) The number of intestinal bacteria is not critical for the enhancement of antitumor activity and reduction of intestinal toxicity of irinotecan by the Chinese herbal medicine PHY906 (KD018). BMC Complement Altern Med 14: 490.

9. Lam W, Jiang Z, Guan F, Huang X, Hu R, et al. (2015) PHY906(KD018), an adjuvant based on an 1800-year-old Chinese medicine, enhanced the antitumor activity of Sorafenib by changing the tumor microenvironment. Sci Rep 5: 9384. 